Letrônica, Porto Alegre, v. 7, n. 1, p. 325-347, jan./jun., 2014

\title{
ESTRATÉGIAS DE EVOCAÇÃO LEXICAL COM CRITÉRIO SEMÂNTICO EM ADULTOS APÓS ACIDENTE VASCULAR CEREBRAL NO HEMISFÉRIO DIREITO
}

\author{
SEMANTIC VERBAL FLUENCY STRATEGIES IN PATIENTS AFTER A \\ RIGHT-HEMISPHERE STROKE
}

\author{
Natalia Becker* \\ Juliana de Lima Müller** \\ Jaqueline de Carvalho Rodrigues ${ }^{* * *}$ \\ Aline Villavicencio ${ }^{* * *}$ \\ Jerusa Fumagalli de Salles ${ }^{* * * * *}$
}

\begin{abstract}
Resumo: Tarefas de fluência verbal semântica (FVS) avaliam a capacidade de gerar palavras a partir de um determinado critério e em um tempo específico, permitindo investigar habilidades como linguagem, memória e funções executivas. 0 objetivo deste estudo foi analisar o desempenho de adultos que sofreram acidente vascular cerebral no hemisfério direito (HD) em uma tarefa de FVS. Foram avaliados 11 adultos com lesão no HD e 11 neurologicamente saudáveis, emparelhados por sexo, idade e escolaridade. Os participantes responderam uma tarefa de FVS (roupas e vestimentas), sendo mensurados o número total de palavras evocadas, variáveis de clustering e switching e a conformação de redes semânticas, por meio de análise computacional. Houve diferenças estatisticamente significativas apenas no número de palavras verbalizadas (escore Z). As análises topográficas de grafos demonstraram tendência a redes semânticas menores no grupo com lesão no HD, em relação aos controles. Análises de série de casos indicaram quatro indivíduos com escores deficitários no número de palavras e na variável switching. Esses participantes apresentavam lesões subcorticais e predominantemente frontais, sugerindo que a localização específica da lesão tem interferência na utilização de estratégias de
\end{abstract}

\footnotetext{
${ }^{*}$ Mestranda do Programa de Pós-Graduação em Psicologia da Universidade Federal do Rio Grande do Sul (UFRGS). Este trabalho é proveniente do projeto Índices para reabilitação neuropsicológica das sequelas de Acidente Vascular Cerebral: aspectos demográficos, neurológicos, neuropsicolinguísticos e de neuroimagem funcional, financiado pelo DECIT/SCTIE/MS, por intermédio do CNPq, apoio da FAPERGS e SES/RS.

${ }^{* *}$ Mestre e Doutoranda pelo Programa de Pós-Graduação em Psicologia da Universidade Federal do Rio Grande do Sul (UFRGS).

*** Mestre e Doutoranda do Programa de Pós-Graduação em Psicologia da Universidade Federal do Rio Grande do Sul (UFRGS).

**** Mestre em Ciência da Computação, Phd em Computer Science e MPhil em Computer Speech and Language Processing. Professora adjunta do Instituto de Informática da Universidade Federal do Rio Grande do Sul, e Erasmus-Mundus Scholar na University of Saarland (Alemanha).

${ }^{* * * * *}$ Mestre e Doutora em Psicologia. Professora adjunta do Instituto de Psicologia, Programa de PósGraduação em Psicologia, Universidade Federal do Rio Grande do Sul (UFRGS). Coordenadora do Núcleo de Estudos em Neuropsicologia Cognitiva (Neurocog).
} 
evocação lexical. Destaca-se o caráter pioneiro deste estudo no contexto brasileiro e a importância da utilização de comparações entre grupos e série de casos em investigações na neuropsicologia cognitiva.

Palavras-chave: Funções executivas; Hemisfério cerebral direito; Análise computacional; Neuropsicologia.

\begin{abstract}
Semantic verbal fluency tasks (SVF) evaluate the ability to generate words from a given criterion in a specified time; these tasks allow for the investigation of language, memory and executive functions. This study aimed to investigate the performance of right hemisphere (RH) post stroke adults in an SVF task. Eleven adults with RH lesion and eleven healthy adults, matched by sex, education and age, were investigated. The participants completed an SVF task (clothes names); the total number of words, clustering and switching variables, and the conformation of semantic networks were measured through computational analysis. We found significant statistical differences in the total number of words (Z score). Topographic analysis of graphs showed a tendency toward smaller semantic networks in the RH lesion group compared with controls. Case series analyzes showed four individuals with deficits in the total number of words and in switching. These participants had predominantly frontal lobe and subcortical lesions, which indicates the interference of lesion-specific locations in the use of SVF strategies. The qualitative analyses presented are groundbreaking for the Brazilian context. We also note the importance of using comparisons between groups and case series analyzes in cognitive neuropsychology investigations.
\end{abstract}

Keywords: Executive functions; Right brain hemisphere; Computational analysis; Neuropsychology.

\title{
Introdução
}

A fluência é um processo executivo caracterizado pela capacidade de um indivíduo realizar uma série de comportamentos dentro de uma estrutura de regras estabelecidas (BENTON, 1968; LEZAK; HOWIESON; LORING, 2004; MALLOY-DINIZ et al., 2010; THURSTONE, 1938), podendo estes serem verbais ou não verbais. Tarefas de fluência verbal (FV), ou de evocação lexical, são amplamente utilizadas em contextos clínicos e experimentais e caracterizam-se por permitirem o acesso aos componentes de habilidades cognitivas como linguagem, memória e funções executivas (LEZAK et al., 2004). Nestas tarefas, o indivíduo é solicitado a gerar o maior número de palavras possível a partir de um determinado critério, em geral durante sessenta segundos (STRAUSS et al., 2006).

Na Fluência Verbal Semântica (FVS) as palavras geradas devem pertencer a uma determinada categoria, como frutas, animais ou peças de vestuário (ARAUJO et al., 2011; BRUCKI et al., 1997; BRUCKI; ROCHA, 2004; CARAMELLI et al., 2007; CHARCHATFICHMAN et al., 2009; LOPES et al., 2009; SILVA et al., 2011). Essas atividades avaliam a velocidade e facilitação da produção verbal e da resposta, organização mental, 
estratégias de busca e habilidade para iniciar um comportamento em resposta a um novo estímulo. Estas características se relacionam aos componentes de volição, flexibilidade e inibição das funções executivas. Além disso, envolvem funções da linguagem, tais como evocação lexical, tamanho do vocabulário e acesso ao léxico, assim como compreendem também as memórias de trabalho e semântica de longo prazo (LEZAK et al., 2004). Do ponto de vista linguístico, tarefas de FVS medem a capacidade de acessar o léxico semântico e recuperar a informação formal e semântica das palavras (JAICHENCO; WILSON; RUIZ, 2007).

$\mathrm{O}$ escore geralmente obtido nos testes de FV é o número total de palavras verbalizadas corretamente pelo indivíduo, comparando esse desempenho com seu grupo normativo, de acordo com a idade e a escolaridade (STRAUSS et al., 2006). No entanto, essa pontuação gera pouca informação sobre os processos cognitivos envolvidos no desempenho da fluência, não respondendo à questão de por que um grupo particular de pacientes apresenta um desempenho deficitário em FV (TROYER, 2000).

Para um bom desempenho nas tarefas de FV é necessário que o respondente gere palavras dentro de uma mesma subcategoria (clustering) e somente troque para a próxima (switching) após ter esgotado todas as palavras da anterior (TROYER, 2000). Os conceitos de clustering e switching foram operacionalizados por Troyer, Moscovitch e Winocour (1997). O primeiro envolve a busca de palavras relacionadas ao critério da tarefa, estando relacionado à memória semântica. Já o componente switching (alternância) envolve habilidades das funções executivas, tais como flexibilidade cognitiva (trocar de uma subcategoria à outra) e inibição.

Em adultos neurologicamente saudáveis já foi demonstrado certo padrão na FV e nas características sociodemográficas que interferem neste processamento. 0 estudo normativo de Troyer (2000) destacou uma redução no número de palavras geradas com o aumento da idade, e em relação às variáveis de clustering e switching: quanto maior a idade do sujeito, maior o tamanho dos clusters e menor o número de switches. No que concerne ao nível educacional, há um baixo efeito desta variável isolada nos componentes de clustering e switching, no entanto, para o número total de palavras evocadas encontrou-se um aumento desta variável com o aumento da escolaridade.

No Brasil, Brucki e Rocha (2004) utilizaram a tarefa de FVS para avaliar os efeitos 
da idade e do nível educacional no desempenho geral e nas variáveis de clustering e switching em uma amostra de brasileiros de 16 a 88 anos. Diferentemente do estudo de Troyer (2000), as autoras não encontraram efeitos de idade, mas sim de escolaridade. Nesta última, também o aumento da escolaridade implicou a melhora do desempenho geral e no aumento do número de clusters. Dessa forma, o aumento da escolaridade facilita o acesso semântico, aparentemente ampliando as possibilidades de repertório categórico (BRUCKI; ROCHA, 2004).

Nos estudos em pacientes com acometimento neurológico, destaca-se um maior comprometimento nas lesões frontais na tarefa de FV, possivelmente devido a uma dificuldade na iniciação e/ou na flexibilidade de busca de palavras e aos processos de retenção (TROYER et al., 1998[a]). Quanto ao desempenho qualitativo, o componente switching também é relacionado ao funcionamento frontal do cérebro. Dessa forma, tal funcionamento mostra-se prejudicado entre os pacientes com lesões na região dorsolateral do hemisfério esquerdo e superior medial do lobo frontal (TROYER et al., 1998[b]), na doença de Parkinson (TRÖSTER et al., 1998; TROYER et al., 1998[b]), na esclerose múltipla (TRÖSTER et al., 1998) e na doença de Huntington (RICH et al., 1999). Em contrapartida, o componente clustering está relacionado ao funcionamento do lobo temporal, avaliado pelo desempenho prejudicado entre pacientes que apresentam comprometimento dessa região cerebral, como na doença de Alzheimer (LOPES et al., 2009; TRÖSTER et al., 1998; TROYER et al., 1998[b]) e nas epilepsias refratárias de lobo temporal (TROYER et al., 1998[a]).

Estudos sobre a lateralidade das lesões têm demonstrado que distúrbios léxicosemânticos são observados em um grande número de indivíduos acometidos por lesões unilaterais no hemisfério cerebral direito (LHD), os quais tendem a produzir menor número de palavras em tarefas de FVS (BEAUSOLEIL et al., 2003; JOANETTE; GOULET; LE DORZE, 1988) e a ativar conexões semânticas periféricas, produzindo palavras que não são fortemente relacionadas (ABUSAMRA et al., 2009). No entanto, ainda permanecem dúvidas quanto às consequências das LHD no desempenho de adultos nessas tarefas (GOULET et al., 1997).

Pesquisas de neuroimagem funcional com adultos neurologicamente saudáveis também convergem para a proposta da participação do hemisfério direito (HD) no processamento semântico (DONNELLY; ALLENDORFER; SZAFLARSKY, 2011), 
demonstrando que a melhor acurácia em uma tarefa de decisão lexical semântica explícita está associada com a ativação do lobo parietal inferior direito e a um maior recrutamento da região frontal direita. Estudos recentes seguem investigando as consequências de lesões na FV; no entanto, não foram encontrados resultados referentes às LHD (FERNÁNDEZ-ANDUJA et al., 2014; PERLAKI et al., 2013).

Por outro lado, um estudo brasileiro que buscou comparar o desempenho de pacientes com LHD e controles, em tarefas de processamento léxico-semântico (efeitos de priming semântico, evocação lexical com critério semântico e julgamento semântico) não encontrou diferenças significativas na comparação entre os dois grupos nas medidas diretas de processamento léxico-semântico (MÜLLER; BECKER; SALLES, 2014, in press). A análise realizada para a tarefa FVS foi apenas de caráter quantitativo, o que não permite a avaliação dos processos subjacentes no desempenho dos grupos.

Outras análises que podem verificar as estratégias de evocação lexical provêm de técnicas e conceitos da informática e da área da computação (ALBERT; BARABASI, 2002). A análise de grafos vem sendo realizada sobre as associações de palavras (STEYVERS; TENENBAUM, 2005; ZORTEA et al., 2014) de modo a propiciar maior compreensão sobre as associações semânticas entre palavras e diferenças no léxicosemântico entre indivíduos neurologicamente saudáveis e com lesões neurológicas.

Nesse contexto, este artigo tem como objetivo examinar os componentes qualitativos e cognitivos de clustering e switching, e a análise de grafos em uma tarefa de avaliação de FVS (roupas e vestimentas) em um grupo de adultos após Acidente Vascular Cerebral (AVC) no HD, comparado a adultos neurologicamente saudáveis (controles). Esperava-se que os pacientes com LHD apresentassem um desempenho inferior ao dos controles para a variável de tamanho médio dos clusters e número de clusters, uma vez que o processamento léxico-semântico, um dos componentes da comunicação que envolve o acesso lexical (DELL et al., 1997; LEVELT; MEYER, 1999), geralmente encontra-se deficitário nos casos com LHD (ABUSAMRA et al., 2009). Esperava-se ainda que uma análise topológica das redes semânticas de cada participante e de cada grupo também refletisse essas diferenças de desempenho. 


\section{Método}

\subsection{Participantes}

Participaram deste estudo 11 adultos com diagnóstico de AVC no HD e 11 adultos neurologicamente saudáveis (controles), emparelhados ao grupo clínico por idade, sexo e escolaridade (Tab. 1). Os pacientes pós-AVC foram recrutados no Ambulatório de Neurovascular do Hospital de Clínicas de Porto Alegre (HCPA) e os participantes do grupo controle foram selecionados na comunidade por amostragem de conveniência. Este estudo teve aprovação do Comitê de Ética em pesquisa do HCPA sob o protocolo número 100149.

Todos os participantes eram destros, monolíngues, falantes do português brasileiro, não apresentavam histórico de diagnóstico psiquiátrico ou neurológico (apenas o AVC, no grupo clínico), nem indícios de quadro depressivo grave, avaliado pelo Inventário Beck de Depressão (BDI- CUNHA, 2001) ou pela Escala de Depressão Geriátrica Yesavage - versão reduzida - GDS-15 (ALMEIDA; ALMEIDA, 1999). Além disso, não faziam uso abusivo e atual de drogas ilícitas ou álcool e não apresentavam dificuldades de visão e audição não corrigidas.

Tabela 1 - Média e desvio-padrão da idade, anos de estudo e hábitos de leitura e escrita dos grupos

\begin{tabular}{lccc}
\hline & $\begin{array}{c}\text { AVC no HD } \\
\text { (n= 11) }\end{array}$ & $\begin{array}{c}\text { Controles } \\
\text { (n= 11) }\end{array}$ & $\boldsymbol{p}^{*}$ \\
\hline Sexo (F/ M) & $6 / 5$ & $6 / 5$ & 0,66 \\
Idade & $54,45(10,79)$ & $54,73(11,74)$ & 0,96 \\
Anos de estudo & $9,55(3,75)$ & $10,91(4,61)$ & 0,34 \\
Hábitos de leitura** & $\begin{array}{c}6,73(3,25) \\
\text { (Baixa Frequência) }\end{array}$ & $\begin{array}{c}8,36(2,83) \\
\text { (Baixa Frequência) }\end{array}$ & 0,15 \\
Hábitos de escrita** & $\begin{array}{c}4,27(3,03) \\
\text { (Baixa Frequência) }\end{array}$ & $\begin{array}{c}4,91(1,9) \\
\text { (Baixa Frequência) }\end{array}$ & $0,01^{*}$ \\
\hline
\end{tabular}

Nota. Diferenças entre os sexos foram analisadas por meio do teste qui-quadrado, enquanto as demais variáveis foram comparadas utilizando o teste $\mathrm{t}$ para amostras independentes. ${ }^{*} p<0,05$. ** Medidos através de escala adaptada dos estudos de Pawlowski (2011) e Pawlowski et al. (2012).

Os participantes do grupo clínico apresentaram único episódio de AVC isquêmico ( 2 pacientes) ou hemorrágico ( 9 pacientes) apenas no HD (confirmado por exames de 
neuroimagem e por avaliações neurológicas), ocorrido há no mínimo seis meses e no máximo 32 meses. Esses casos não manifestaram alterações de linguagem semelhantes a quadro de afasia (avaliados por tarefas de linguagem oral e escrita do Instrumento de Avaliação Neuropsicológica Breve (NEUPSILIN et al., 2009). No grupo controle, incluíram-se adultos sem indícios de comprometimento cognitivo ou demência (Mini Exame do Estado Mental - MEEM; FOLSTEIN; FOLSTEIN; MCHUGH, 1975; versão adaptada por CHAVES; IZQUIERDO, 1992; KOCHHANN et al., 2010). Ambos os grupos assinaram um Termo de Consentimento Livre e Esclarecido e em seguida realizaram a avaliação neuropsicológica que ocorreu individualmente com duração aproximada de 45 minutos.

\subsection{Materiais e procedimentos}

A avaliação da FV foi realizada por meio da tarefa de evocação lexical com critério semântico da Bateria Montreal de Avaliação da Comunicação - Bateria MAC (FONSECA et al., 2008). Os participantes foram solicitados a evocar o maior número possível de palavras que fossem roupas ou vestimentas durante dois minutos. Considerou-se erro palavras repetidas ou que não pertencessem à categoria, conforme o manual do instrumento.

Para a análise quantitativa, verificou-se o número total de palavras corretas evocadas durante o primeiro e o segundo minuto, considerando-se erros repetições e palavras não pertencentes ao critério semântico estabelecido. Os componentes qualitativos de clustering e switching foram identificados por meio de dois escores, conforme proposto por Troyer et al. (1997) e adaptado por Lopes et al. (2009): (1) média do tamanho dos conjuntos de subcategorias (clusters), (2) número de trocas de subcategorias (switches). A média do tamanho dos conjuntos foi obtida somando-se as palavras de uma mesma subcategoria (cluster) a partir da segunda palavra. A média do tamanho do cluster foi calculada somando-se o tamanho de cada cluster dividido pelo número de clusters. 0 número de trocas de subcategorias (switches) foi calculado pela soma do número de trocas entre clusters, incluindo também palavras isoladas. Erros e repetições foram incluídos na análise qualitativa, pois proporcionam informações sobre os processos cognitivos empregados pelos participantes (TROYER et al., 1997; TROYER, 
2000). Todos os protocolos foram pontuados por dois juízes de forma independente, operacionalizando-se as subcategorias. Um terceiro juiz analisou as variáveis em que não houve concordância entre os dois primeiros juízes. Maiores informações sobre o processo de operacionalização destas variáveis encontram-se no Anexo deste artigo.

Além disso, foram construídas redes semânticas individuais para os participantes de cada grupo, e para o grupo como um todo. As redes dos indivíduos são formadas por nós que correspondem a cada uma das diferentes palavras nomeadas, e dois nós, $a$ e $b$, estão ligados através de uma aresta direcionada se a palavra a foi nomeada imediatamente antes da palavra $b$, figura 1. Quando há uma repetição, essa faz com que um laço seja formado voltando ao ponto da primeira ocorrência da palavra, como, por exemplo, na sequência mostrada na figura $1, a, b, c, d, e, b, f$. No caso das redes, foram mantidas as repetições para calcular qual a incidência média por grupo e se elas ocorriam muito próximas uma da outra (ciclos de 1, 2 ou 3 nós). Foram usadas redes direcionadas para medir a recorrência com que uma determinada palavra evocava uma outra numa certa ordem (ex. calça $\rightarrow$ camisa, pão $\rightarrow$ manteiga). As redes individuais dos participantes foram usadas como base para caracterizar a topologia média de cada um dos grupos.

Figura 1: Exemplos de redes direcionadas de dois indivíduos

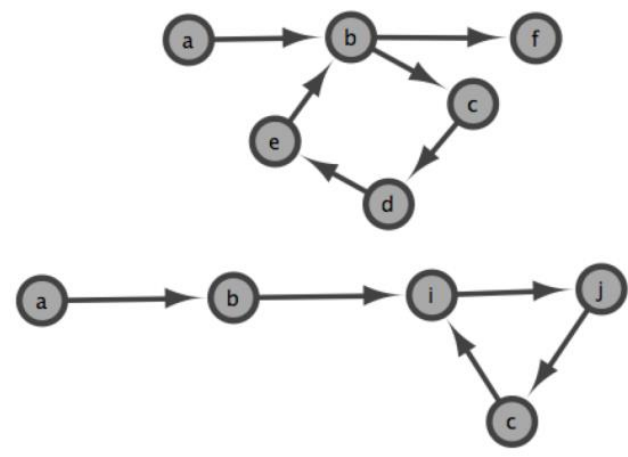

Adicionalmente para cada grupo foi gerada também uma rede semântica nãodirecionada contendo as palavras nomeadas por todos os participantes do grupo. Uma aresta não-direcionada é usada para ligar dois nós se eles correspondem a palavras que foram nomeadas em sequência por algum dos participantes. As redes dos indivíduos da figura 1 formam a rede da figura 2. 
Figura 2: Exemplo de rede não-direcionada dos participantes de um grupo

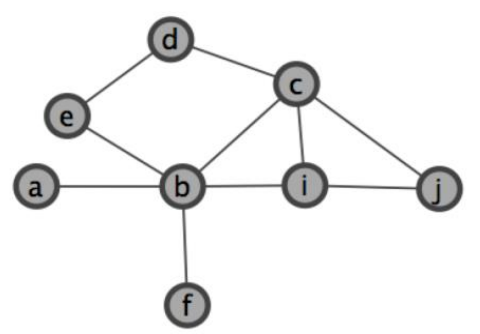

As redes foram caracterizadas por algumas variáveis topológicas (ALBERT; BARABÁSI, 2002), tais como o número total de nós, o número total de arestas, coeficiente de clusterização, grau médio dos nós, grau total médio, densidade e diâmetro da rede e a média do menor caminho entre dois nós da rede, usadas para diferenciar redes de palavras (STEYVERS; TENENBAUM, 2005) (Quadro 1). As comparações entre os grupos foram feitas tanto em termos da topologia média das redes individuais dos participantes de cada grupo, como em termos da topologia da rede global formada por todos os participantes de um grupo.

Quadro 1: Medidas topológicas para descrever as redes semânticas

\begin{tabular}{|l|l|}
\hline Medida & Descrição \\
\hline Total de nós & número de palavras usadas \\
\hline Total de arestas & número total de ligações entre os nós na rede \\
\hline Grau total médio & $\begin{array}{l}\text { grau médio dos nós da rede, sendo que o grau de um nó é a soma de todas } \\
\text { as suas arestas }\end{array}$ \\
\hline Densidade & número de arestas dividido pelo número de possíveis arestas \\
\hline Diâmetro & tamanho do maior caminho mais curto entre dois nós da rede \\
\hline $\begin{array}{l}\text { Coeficiente } \\
\text { clusterização de }\end{array}$ & $\begin{array}{l}\text { coeficiente de clusterização médio dos nós da rede, sendo que o } \\
\text { coeficiente de um nó mede o número de vizinhos dele que também são } \\
\text { vizinhos entre si }\end{array}$ \\
\hline $\begin{array}{l}\text { Média do Menor } \\
\text { Caminho }\end{array}$ & $\begin{array}{l}\text { número médio de passos no menor caminho entre dois nós quaisquer da } \\
\text { rede }\end{array}$ \\
\hline
\end{tabular}

\section{Análises dos dados}

As variáveis dependentes apresentaram distribuição normal (Teste de Shapiro- 
Wilk) em ambos os grupos. A média do tamanho dos clusters, o número de switches e o número total de palavras corretas evocadas foram analisados por um teste $\mathrm{t}$ de Student $(p \leq 0,05)$ de medidas independentes para uma análise entre os grupos. Na análise topológica para cada grupo foram avaliadas as médias de cada uma das medidas das redes individuais, também com o teste $t$.

Realizaram-se análises de série de casos (SCHWARTZ; DELL, 2010) com os participantes que apresentaram desempenhos deficitários na tarefa de Evocação Lexical com Critério Semântico da Bateria MAC (FONSECA et al., 2008), a fim de identificar igualdades e semelhanças nos mecanismos cognitivos subjacentes à habilidade de FV. Consideraram-se deficitários os escores iguais ou menores a -1,5 desvios-padrão em relação à amostra normativa da Bateria MAC (FONSECA et al., 2008).

\subsection{Resultados}

Os resultados da Tarefa de Evocação Lexical com critério semântico encontramse na tabela 2. 0 teste $t$ de Student para amostras independentes não indicou diferenças significativas entre os grupos em nenhuma das variáveis mensuradas, o que indica um desempenho similar entre pacientes com lesão cerebral de hemisfério direito (LHD) e controles neurologicamente saudáveis. 0 número de switches chegou a ser menor para o grupo de pacientes, porém essa diferença não foi significativa $(\mathrm{t}=-1,52 ; p<0,14)$, possivelmente devido às variâncias intra-grupos serem maiores para essa variável (Desvio padrão - DP=2,82 e 4,06; para os grupos clínico e controle, respectivamente).

De modo a verificar se os grupos não se diferenciavam no número total de palavras evocadas, os escores brutos foram convertidos em escores padronizados (Escores Z) baseados nas médias das tabelas normativas da Bateria MAC (FONSECA et al., 2008). Realizando um novo teste $t$ comparando os escores $\mathrm{Z}$ do número total de palavras evocadas, foram encontradas diferenças significativas $(\mathrm{t}=-2,25 ; p=0,03)$ entre 0 desempenho do grupo LHD $(Z=-1,14 ; D P=0,65)$ e o do grupo de controles $(Z=-0,48$; $\mathrm{DP}=0,71$ ), embora o grupo de pacientes não tenha apresentado um escore $\mathrm{Z}$ indicativo de déficit $(Z<-1,5)$. 
Tabela 2 - Resultados (média e desvio-padrão) dos escores de número total de palavras geradas, tamanho dos clusters, número de clusters e número de switches

\begin{tabular}{lcccccc}
\hline & $\begin{array}{c}\text { AVC no HD } \\
(\boldsymbol{n = 1 1 )}\end{array}$ & \multicolumn{6}{c}{$\begin{array}{c}\text { Controle } \\
(\mathbf{n = 1 1 )}\end{array}$} \\
& $M$ & $D P$ & $M$ & $D P$ & $\mathrm{t}$ & $p$ \\
& & & & & & \\
Número palavras & 17,45 & 4,05 & 21,91 & 6,47 & $-1,93$ & 0,06 \\
$\begin{array}{l}\text { Média tamanho } \\
\text { clusters }\end{array}$ & 1,81 & 0,57 & 1,97 & 0,72 & $-0,58$ & 0,56 \\
Número switches & 7,81 & 2,82 & 10,09 & 4,06 & $-1,52$ & 0,14 \\
\hline
\end{tabular}

Nota. AVC = acidente vascular cerebral; HD = hemisfério direito; Ev. lex. sem. = evocação lexical com critério semântico; $M$ = média; $\mathrm{DP}$ = Desvio padrão.

As redes semânticas geradas refletiram a sequência de evocação de palavras de cada indivíduo e foram analisadas medidas que caracterizaram a topologia média das redes de cada grupo. As figuras 3 e 4 apresentam a conformação das redes. Em termos de tamanho das redes, as do grupo controle têm em média mais nós $(M=23,36)$ do que o grupo com LHD ( $M=19,45)$, são mais longas (diâmetro $M=19,18 \times 15,36$ ) e mais conectadas (arestas $M=22,91 \times 19,27$ ) (Tab. 3), refletindo o número maior de palavras evocadas pelo grupo controle, porém as diferenças não são estatisticamente significativas.

Tabela 3 - Resultados das medidas topológicas médias das redes semânticas dos indivíduos de cada grupo

\begin{tabular}{lllllll}
\hline Rede Semântica & AVC no HD & \multicolumn{3}{l}{ Controle } \\
& M & DP & M & DP & $p^{*}$ \\
Número de nós & 19,45 & 3,78 & 23,36 & 5,08 & 0,05 \\
Número de arestas & 19,27 & 4,13 & 22,91 & 5,11 & 0,08 \\
Grau Total Médio & 1,97 & 0,10 & 1,96 & 0,07 & -- \\
Densidade & 0,11 & 0,02 & 0,09 & 0,02 & 0,05 \\
Diâmetro & 15,36 & 5,05 & 19,18 & 7,25 & 0,16 \\
Média do Menor Caminho & 5,90 & 1,61 & 7,15 & 2,33 & 0,15 \\
Coeficiente de Clusterização & 0 & 0 & 0 & 0 & -- \\
\hline
\end{tabular}

*Nota. Comparações realizadas por Teste t. 
A rede semântica dos grupos também refletiu a maior fluência do grupo controle, com maior número de nós e arestas, que são mais distribuídas pela rede (densidade menor e maior coeficiente de clusterização). No grupo clínico algumas palavras concentravam um maior número de associações, mas as diferenças não foram significativas (Tab. 4 e Fig. 3 e 4).

Tabela 4 - Resultados das medidas topológicas das redes semânticas dos grupos

\begin{tabular}{lrr}
\hline & AVC no HD & Controle \\
\hline Número de Nós & 64 & 84 \\
Número de Arestas & 170 & 211 \\
Grau total médio & 5.0625 & 4.761904762 \\
Densidade & 6 & 0.057372347 \\
Diâmetro & 0.080357143 & 8 \\
Média do Menor Caminho & 2.850198413 & 3.119047619 \\
Coeficiente de Clusterização & 0.122178603 & 0.113585224 \\
\hline
\end{tabular}

Figura 3: Rede semântica do grupo controle na tarefa de evocação lexical. Cores dos nós representam os diferentes clusters definidos

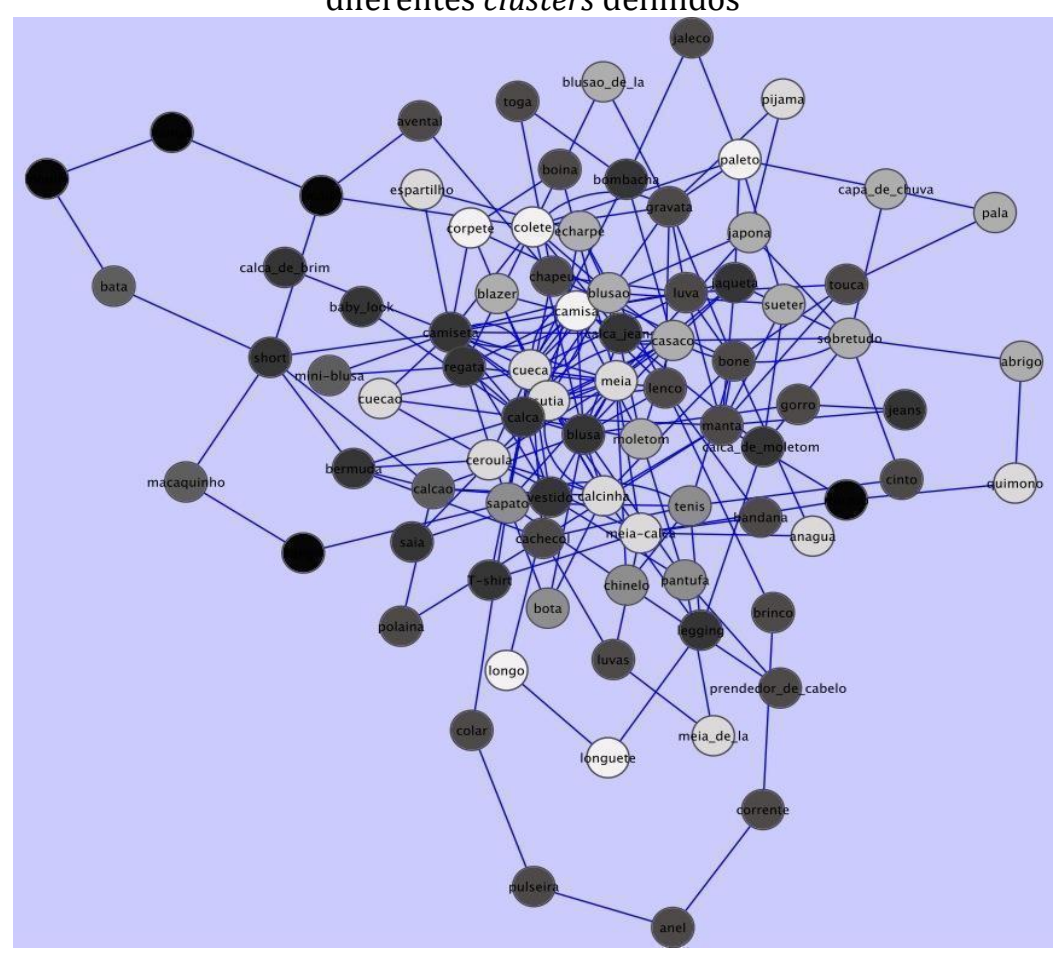


Figura 4: Rede semântica do grupo clínico na tarefa de evocação lexical. Cores dos nós representam os diferentes clusters definidos

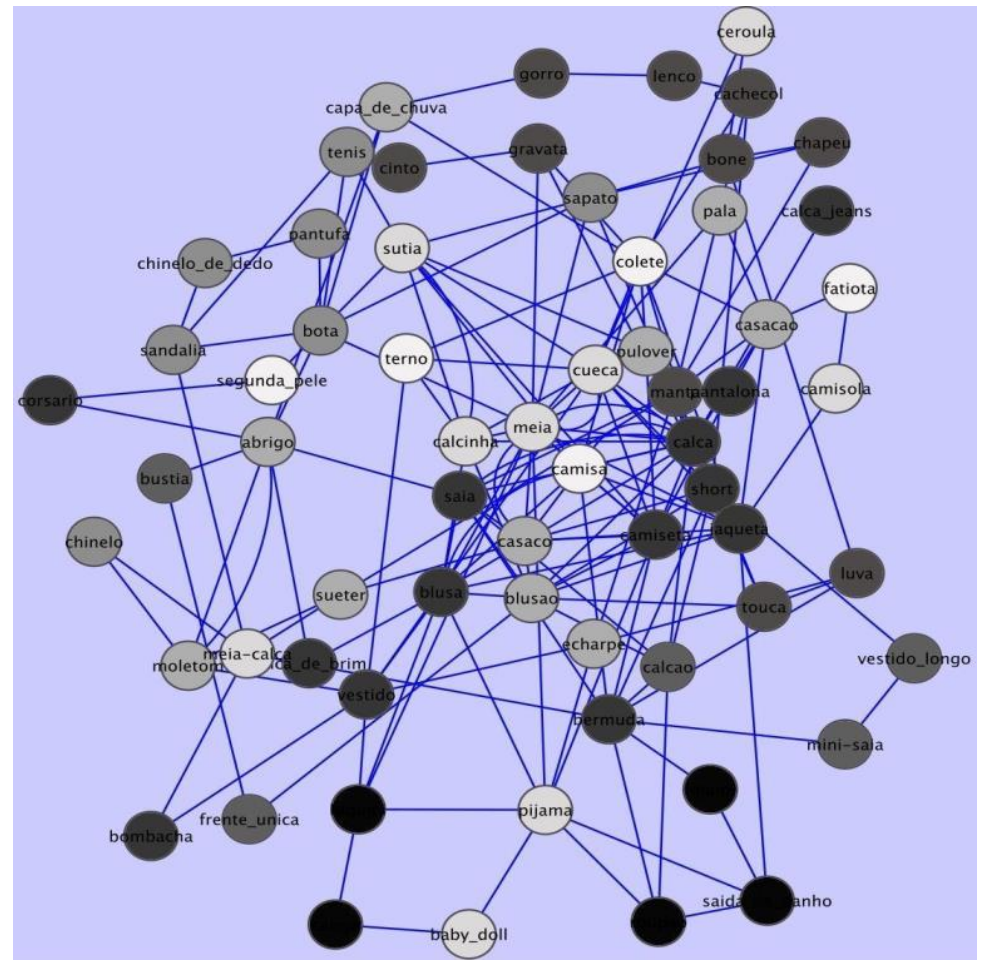

\subsection{Análise de Série de Casos}

Identificaram-se quatro casos (P01, P02, P05 e P07) com desempenho deficitário no número total de palavras evocadas na tarefa de FVS. Consideraram-se déficits escores iguais ou abaixo de -1,5 desvios-padrão da média do grupo normativo (Escore Z) da Bateria MAC (FONSECA et al., 2008). A Tab. 5 apresenta as características da lesão e os desempenhos nas variáveis qualitativas de cada um dos quatro casos.

Tabela 5 - Região e local específico da lesão e os escores nas variáveis de clustering e switching dos pacientes com desempenhos deficitários na Tarefa de Evocação Lexical

\begin{tabular}{cccccc}
\hline Caso & $\begin{array}{c}\text { Local da } \\
\text { lesão }\end{array}$ & $\begin{array}{c}\text { Local específico da } \\
\text { lesão }\end{array}$ & $\begin{array}{c}\text { Escore Z } \\
\mathbf{N}^{\circ} \text { total de } \\
\text { palavras* }\end{array}$ & $\begin{array}{c}\text { Média } \\
\text { tamanho } \\
\text { clusters }\end{array}$ & $\begin{array}{c}\text { Número } \\
\text { switches }\end{array}$ \\
\hline P01 & Subcortical & frontal & $-1,6$ & 3,00 & 4 \\
P02 & Subcortical & fronto-têmporo-parietal & $-1,9$ & 1,22 & 12 \\
\hline
\end{tabular}


Tabela 5 (Continuação)- Região e local específico da lesão e os escores nas variáveis de clustering e switching dos pacientes com desempenhos deficitários na Tarefa de Evocação Lexical

\begin{tabular}{cccccc}
\hline Caso & $\begin{array}{c}\text { Local da } \\
\text { lesão }\end{array}$ & $\begin{array}{c}\text { Local específico da } \\
\text { lesão }\end{array}$ & $\begin{array}{c}\text { Escore Z } \\
\mathbf{N}^{\circ} \text { total de } \\
\text { palavras* }\end{array}$ & $\begin{array}{c}\text { Média } \\
\text { tamanho } \\
\text { clusters }\end{array}$ & $\begin{array}{c}\text { Número } \\
\text { switches }\end{array}$ \\
\hline P05 & Subcortical & tálamo & $-1,890$ & 1,5 & 3 \\
P07 & Subcortical & $\begin{array}{c}\text { substância branca } \\
\text { periventricular }\end{array}$ & $-1,740$ & 1 & 8 \\
\hline
\end{tabular}

*Nota: de acordo com dados normativos da Bateria MAC (FONSECA et al., 2008).

Os demais sete casos clínicos obtiveram Escores Z próximos de zero, indicando desempenhos similares ao grupo normativo. Quatro indivíduos apresentaram lesões corticais (P04, P08, P10, P11) e predominantemente fronto-temporais. Três dos destes casos apresentavam alta frequência de hábitos de leitura e de escrita (P06, P10, P11) e nove ou mais anos de estudos formais.

\section{Discussão}

O objetivo deste estudo foi comparar o desempenho de pacientes com LHD e indivíduos neurologicamente saudáveis em uma tarefa de evocação lexical com critério semântico, a partir dos componentes qualitativos de clustering e switching e da análise topográfica de grafos. Pela comparação entre grupos não houve diferenças significativas nos desempenhos tanto para as variáveis qualitativas quanto na análise de grafos, sugerindo que pacientes com LHD apresentem certo balanceamento dos componentes de clustering e switching, o que é essencial para um bom desempenho na tarefa de FVS (TROYER et al., 1997).

Ressalta-se que, embora não tenham sido encontradas diferenças significativas nessas variáveis, quando comparados os desempenhos dos grupos pelo escore Z, houve diferenças significativas. Este segundo resultado indica que o grupo clínico obteve um desempenho diminuído em relação ao grupo controle, mas que não chegou a prejudicar a fluência em termos de déficit.

Essas análises levam a uma primeira conclusão de que LHD não compromete o acesso lexical, quando avaliado por uma tarefa de FV com categoria de roupas e considerando as características da amostra clínica e da metodologia utilizada. Esse 
resultado deve ser observado com cautela, tendo em vista o reduzido número de participantes em cada grupo e a heterogeneidade intra-grupo. Embora este estudo não tenha encontrado diferenças entre os grupos clínico e controle para as variáveis de clustering e switching, diversos estudos as encontraram (DONOVAN et al., 1999; TRÖSTER et al., 1998; TROYER et al., 1998[a]; TROYER et al., 1998[b]) no sentido de grupos clínicos apresentarem um destes componentes prejudicados, interferindo no desempenho geral da tarefa.

Diferenças metodológicas importantes são encontradas naqueles estudos em relação a este, como o tipo de critério semântico utilizado na tarefa e o tempo de execução. Neste estudo, utilizamos a categoria semântica de roupas ou peças de vestuário, porém os estudos que avaliam os componentes de clustering e switching geralmente utilizam mais de uma categoria, sendo estas animais, frutas ou itens de supermercado (RAOUX et al., 2008; TRÖSTER et al., 2008; TROYER, 2000; TROYER et al., 1997). Estas categorias permitem a ativação de redes semânticas amplas, as quais podem ativar palavras mais fortemente associadas ao conceito, assim como palavras mais distantes. Quanto à amostra clínica, usualmente separam os participantes por local específico da lesão ou por patologias específicas como demência de Alzheimer, demência de Parkinson e doença de Huntington.

Embora sem diferenças significativas entre os grupos, os resultados aqui apresentados demonstram que o grupo de pacientes LHD obteve um desempenho inferior na variável switching que seus controles, o que foi ao encontro da hipótese inicial. Esperava-se que o grupo clínico apresentasse um desempenho inferior em clustering, devido a este componente relacionar-se ao processamento léxico-semântico e, portanto, ao acesso lexical - componente da comunicação normalmente prejudicado em LHD (ABUSAMRA et al., 2009; GOULET et al., 1999).

Estudos que investigaram as habilidades léxico-semânticas através de tarefas de fluência verbal encontraram desempenho geral diminuído nos indivíduos com LHD comparado às pessoas sem lesão cerebral. Contudo, nestas habilidades o prejuízo é menos significativo que o encontrado em lesões de hemisfério esquerdo (LHE) (FONSECA et al., 2007), já que, usualmente, este processamento está mais prejudicado nestes pacientes. 0 estudo dos diferentes níveis de processamento linguístico sugere, ainda, que quanto maior o grau de demanda semântica e/ou lexical, maior estará sendo 
a contribuição do HD. Essas definições corroboram resultados deste estudo a partir da comparação entre os grupos pelo escore $\mathrm{Z}$ para o número total de palavras geradas. Foram encontradas diferenças significativas de desempenho entre os grupos, embora os pacientes LHD não tenham apresentado um escore $Z$ deficitário $(Z=-1,14)$ comparado a amostra normativa do manual do instrumento (FONSECA et al., 2008). Isso demonstra que, possivelmente, devido aos participantes clínicos do estudo estarem com o HE íntegro, a lesão do HD não chegou a prejudicar o desempenho geral em termos de déficit, apenas lentificou a produção das palavras em uma mesma categoria semântica. Além disso, o tempo maior de duração da tarefa (dois minutos) em relação a outros estudos (um minuto) pode ter influenciado na baixa diferença entre os grupos em termos de produção das palavras. 0 grupo clínico pode ter se beneficiado do tempo estendido, e comparações entre os quartis de tempo na tarefa poderiam indicar diferenças.

A partir da análise topográfica de grafos, constatou-se uma diferença qualitativa na fluência dos dois grupos, com os participantes controles tendendo a apresentar redes semânticas mais amplas, com maior número de arestas e nós. Esses resultados corroboram evidências de cooperação inter-hemisférica (FONSECA et al., 2009), já que o HD estaria auxiliando na ampliação das redes semânticas e consequentemente no acesso léxico-semântico. Além disso, a ativação de itens associados menos fortemente ao conceito é relacionada ao funcionamento íntegro do HD (DONNELLY et al., 2011), o que associa-se a conformação dos grafos de cada grupo (Fig. 3 e 4).

Fonseca et al. (2007) apontam que a inclusão de indivíduos com diferentes características neurológicas, como os locais das lesões e diferentes severidades, pode estar comprometendo as análises de estudo de grupos. Nos casos de LHD, o que essas autoras ressaltam é que alguns indivíduos podem estar apresentando déficits comunicativos enquanto outros não. Nesse sentido, torna-se importante a realização de estudos de série de casos na Neuropsicologia Cognitiva, pois proporcionam o desenvolvimento de teorias (modelos de processamento) que abarcam a diversidade dos déficits (CARAMAZZA; COLTHEART, 2006), além de permitir a investigação de subgrupos, através das associações e dissociações de desempenho. Em função disso, enfatiza-se a necessidade de estudos que utilizem delineamento de séries de casos para o melhor entendimento de alterações neuropsicológicas em pacientes acometidos pelo AVC no HD. 
$\mathrm{Na}$ análise de série de casos, observou-se que os quatro pacientes com desempenho deficitário na tarefa de FV apresentavam lesões em regiões anteriores do cérebro. Verificando-se as regiões cerebrais específicas do HD atingidas pelo AVC neste grupo, havia três pacientes com lesões na região frontal do cérebro, a qual é usualmente relacionada ao componente cognitivo de switching. Troyer et al. (1998[a]) encontram resultados similares quando comparam pacientes com lesões frontais e temporais a controles. Os pacientes com lesões frontais à esquerda ou à direita, apresentam desempenho prejudicado no número de switchings na fluência semântica, em relação aos participantes sem lesão. Esses achados levam à conclusão de que estes componentes cognitivos podem estar mais associados à região cerebral da lesão do que a sua lateralidade, por isso esses processos específicos não estariam afetados no desempenho geral do grupo clínico.

Outra característica interessante a ser observada é que os quatro casos com desempenho deficitário na tarefa (conforme norma do instrumento) apresentaram lesões subcorticais. Estudos envolvendo a análise dos componentes de clustering e switching em doenças como Parkinson e Huntington (subcorticais) também referem déficits no componente de switching, o que prejudica o desempenho geral na tarefa (RICH et al., 1999; TROYER et al., 1998[a]). Estudos futuros podem comparar grupos de pacientes com lesões corticais e subcorticais de modo a melhor investigar o impacto da localização da lesão nestas estratégias.

\section{Considerações finais}

O objetivo principal desta pesquisa foi investigar o papel do HD no desempenho em tarefas de fluência verbal semântica, a partir dos componentes qualitativos de clustering e switching e da análise topográfica de grafos. Ao contrário das expectativas, os indivíduos LHD apresentaram desempenho similar ao dos participantes sem lesão cerebral em ambos os componentes, não sendo encontradas diferenças significativas entre os grupos nestas variáveis. Contudo, o grupo clínico apresentou desempenho inferior em relação ao grupo controle no componente switching, o que pode ser explicado pela maior parte dos indivíduos apresentarem lesões em regiões frontais (TROYER et al., 1997). Além disso, na análise de série de casos observou-se que os casos 
com lesões anteriores mostraram desempenho deficitário na tarefa de FVS, corroborando estudos que mostram a importância dos lobos frontais e temporais nesse processamento.

0 presente estudo representa uma investigação dos processamentos subjacentes à fluência verbal semântica em indivíduos pós-AVC no HD pioneira no contexto brasileiro. Estes resultados devem ser visualizados com cautela, tendo em vista algumas limitações metodológicas discutidas anteriormente, como número reduzido de participantes, a utilização de apenas um tipo de tarefa de fluência semântica, a não inclusão de tarefa de fluência verbal fonêmica e a variabilidade de regiões das lesões no grupo clínico. Salienta-se a importância da união entre estudos de grupo e série de casos (SCHWARTZ; DELL, 2010) nas investigações que utilizam a abordagem em neuropsicologia cognitiva, já que esta segunda modalidade permite pensar hipóteses clínicas a partir de modelos teóricos (RAPP, 2011). Assim, este estudo não permite a generalização dos achados para a população em geral, mas propõe um panorama inicial sobre a influência do HD no desempenho de tarefas de FV, a partir de componentes qualitativos.

\section{Referências}

ABUSAMRA, Valeria et al. Communication impairments in patients with right hemisphere damage. Life Span and Disabilities, v. 12, n. 1, p. 67-82, 2009.

ALBERT, Réka; BARABÁSI, Albert. Statistical mechanics of complex networks. Reviews of Modern Physics, v. 74, p. 47-97, 2002.

ALMEIDA, Osvaldo; ALMEIDA, Shirley. Confiabilidade da versão brasileira da escala de depressão geriátrica (GDS) versão reduzida. Arquivos de Neuropsiquiatria, v. 57, n. 2-B, p. 421-426, 1999.

ARAUJO, Narahyana et al. Verbal Fluency in Alzheimer's disease, Parkinson's disease and major depression. Clinics, v. 66, n. 4, p. 623-627, 2011.

BEAUSOLEIL, Natacha; FORTIN, Brigitte; JOANETTE, Yves. Unconstrained oral naming performance in right-and left-hemisphere-damaged individuals: When education overrides the lesion. Aphasiology, v. 17, p. 143-158, 2003.

BENTON, Arthur. Differential behavioral effects in frontal lobe disease. Neuropsychologia, v. 6, p. 53-60, 1968. 
BRUCKI, Sonia et al. Dados normativos para o teste de fluência verbal categoria animais em nosso meio. Arquivos de Neuropsiquiatria, v. 55, n. 1, p. 56-61, 1997.

BRUCKI, Sonia; ROCHA, Maria. Category fluency test: effects of age, gender and education on total scores, clustering and switching in Brazilian Portuguese-speaking subjects. Brazilian Journal of Medical and Biological Research, v.37, p. 1771-1777, 2004.

CARAMAZZA, Afonso; COLTHEART, Max. Cognitive Neuropsychology twenty years on. Cognitive Neuropsychology, v. 23, n. 1, p. 3-12, 2006.

CARAMELLI, Paulo et al. Category fluency as a screening test for Alzheimer disease in illiterate and literate patients.Alzheimer Disease Associations Disroders, v. 21, n. 1, p. 6567, 2007.

CHARCHAT-FICHMAN, Helenice et al. Age and educational level effects on the performance of normal elderly on category fluency tasks. Dementia \& Neuropsychologia, v. 3, n. 1, p. 49-54, 2009.

CHAVES, Marcia; IZQUIERDO, Ivan. Differential diagnosis between dementia and depression: a study of efficiency increment. Acta Neurologica Scandinavia, v. 11, p. 412429, 1992.

CUNHA, Jurema. Escalas Beck. São Paulo: Casa do Psicólogo, 2001.

DELL, Gary et al. Lexical Access in Aphasic and Nonaphasic Speakers. Psychological Review, v. 104, n. 4, p. 801-838, 1997.

DONNELLY, Kielly; ALLENDORFER, Jane; SZAFLARSKI, Jerzy. Right hemispheric participation in semantic decision improves performance. Brain Research, v. 1419, p. 105-116, 2011;

DONOVAN, Kelly et al. Clustering and switching in verbal fluency in Parkinson's Disease. New Zealand Journal of Psychology, v. 28, n. 1, p. 61-66, 1999.

FERNÁNDEZ-ANDÚJAR, Marina et al. Thalamic diffusion differences related to cognitive in white matter lesions. Neurobiology of Aging, v. 35, n. 5, p. 1103-1110., 2014.

FOLSTEIN, Marshal; FOLSTEIN, Susan; MCHUGH, Paul. Mini-mental state. Journal of Psychiatry Resources, v. 12, p. 189-198, 1975.

FONSECA, Rochele et al. Right hemisphere damage: communication processing in adults evaluated by the Brazilian Protocole MEC Bateria MAC. Dementia \& Neuropsychologia, v. 1, p. 266-275, 2007.

FONSECA, Rochele et al. Bateria Montreal de Avaliação da Comunicação - Bateria MAC. São Paulo: Pró-Fono, 2008.

FONSECA, Rochele; SALLES, Jerusa; PARENTE, Maria. Instrumento de Avaliação 
Neuropsicológica Breve Neupsilin. São Paulo: Vetor Editora, 2009.

FONSECA, Rochele et al. Hemispheric specialization for communicative processing: neuroimaging data on the role of right hemisphere. Psychology \& Neuroscience, v. 2, n. 1, p. 25-33, 2009.

GOULET, Pierre et al. Word fluency after a right-hemisphere lesion. Neuropsychologia, v. 35, p. 1565-1570, 1997.

JAICHENCO, Virginia; WILSON, Maximiliano; RUIZ, Adelaide. Evaluación del lenguaje. In: BURIN, Debora I.; DRAKE, M. A; HARRIS, P. (Orgs.). Evaluación neuropsicológica en adultos. Buenos Aires: Paidós, 2007, p. 213-241.

JOANETTE, Yves; GOULET, Pierre. Criterion-specific of verbal fluency in right brain damage right-handers. Neuropsychologia, v. 24, p. 875-879, 1986.

KOCHHANN, Renata et al. The Mini Mental State Examination Review of cutoff points adjusted for schooling in a large Southern Brazilian sample. Dementia \& Neuropsychologia, v. 4, n. 1, p. 35-41, 2010.

LEVELT, Willem; MEYER, Antje. A theory of lexical access in speech production. Behavioral and Brain Sciences, v. 22, p. 1-75, 1999.

LEZAK, Muriel; HOWIESON, Diane; LORING, David. Neuropsychological Assessment. Oxford: Oxford University Press, 2004.

LOPES, Marcos. et al. Semantic Verbal Fluencytest in dementia: preliminary retrospective analysis. Dementia \& Neuropsychologia, v. 3, n. 4, p. 315-320, 2009.

MALLOY-DINIZ, Leandro et al. Exame das Funções Executivas. In: MALLOY-DINIZ, Leandro F. (Org.). Avaliação Neuropsicológica. Porto Alegre: Artmed, 2010. p. 94-113.

MÜLLER, Juliana; BECKER, Natalia; SALLES, Jerusa. Desempenho de pacientes com lesão no hemisfério cerebral direito em tarefas de processamento léxico- semântico. Ciências $e$ Cognição, 2014, in press.

PAWLOWSKI, Josiane. Instrumento de avaliação neuropsicológica breve Neupsilin: evidências de validade de construto e de validade incremental à avaliação neurológica. 2011. 139 f. Tese (Doutorado em Psicologia) - Programa de Pós-Graduação em Psicologia, Instituto de Psicologia, Universidade Federal do Rio Grande do Sul, Porto Alegre, Brasil.

PAWLOWSKI, Josiane et al. The influence of reading and writing habits associated with education on the neuropsychological performance of Brazilian adults. Reading and Writing, v. 25, p. 2275-2289, 2012.

PERLAKI, Gabor et al. White-matter microestructure and language lateralization in lefthanders: A whole brain MRI study. Brain and Cognition, v. 82, n. 3, p. 319-328, 2013. 
RAOUX, Nadine et al. Clustering and switching processes in semantic verbal fluency in the course of Alzheimer's disease subjects: results from the PAQUID longitudinal study. Cortex, v. 44, p. 1188-1196, 2008.

RAPP, Brenda. Case series in cognitive neuropsychology: Promise, perils, and proper perspective. Cognitive neuropsychology, v. 28, n. 7, p. 435 -444, 2011.

RICH, Jill et al. Longitudinal analysis of phonemic clustering and switching during word list generation in Huntington's disease. Neuropsychology, v. 13, p. 525-531, 1999.

SCHWARTZ, Myrna; DELL, Gary. Case series investigations in cognitive neuropsychology. Cognitive Neuropsychology, v. 27, n. 6, p. 477-494, 2010.

SILVA, Thaís et al. Fluência Verbal e variáveis sócio-demográficas no processo de envelhecimento: Um estudo epidemiológico. Psicologia: Reflexão e Crítica, v. 24, n. 4, p. 739-746, 2011.

STEYVERS, Mark; TENENBAUM, Joshua. The large-scale structure of semantic networks: Statistical analyses and a model of semantic growth. Cognitive Science, v. 29, n. 1, p. 4178, 2005.

STRAUSS, Esther; SHERMAN, Elizabeth; SPREEN, Otfried. A compendium of neuropsychological tests: Administration, norms and commentary. New York: Oxford University Press, 2006.

THURSTONE, Louis Leon. Primary mental abilities. Psychometric Monographs, v. 1, 1938.

TRÖSTER, Alexsander et al. Cortical and subcortical influences on clustering and switching in the performance of verbal fluency tasks. Neuropsychologia, v. 36, n. 4, p. 295-304, 1998.

TROYER, Angela. Normative data for Clustering and Switching on Verbal Fluency Tasks. Journal of Clinical and Experimental Neuropsychology, v. 22, n. 3, p. 370-378, 2000.

TROYER, Angela; MOSCOVITCH, Morris; WINOCUR, Gordon. Clustering and Switching as two components of Verbal Fluency: evidence from younger and older healthy adults. Neuropsychology, v. 11, n. 1, p. 138-136, 1997.

TROYER, Angela et al. Clustering and Switching on verbal fluency: the effects of focal frontal- and temporal -lobe lesions. Neuropsychologia, v. 36, n. 6, p. 499-504, 1998[a].

TROYER, Angela et al. Clustering and Switching on verbal fluency tests in Alzheimer's and Parkinson's disease. Journal of the International Society, v. 4, p. 137-143, 1998[b].

ZORTEA, Maxciel et al. Graph Analysis of Semantic Word Association between Children, Adults, and the Elderly. Psicologia: Reflexão e Crítica (Impresso), v. 27, n. 1, p. 1-10, 2014. 
Recebido em março de 2014.

Aceito em junho de 2014.

\begin{abstract}
Anexo I
Regras para classificação de clusters em fluência semântica - categoria roupas/peças de vestimenta - baseado nos critérios de categorização de Troyeret al. (1997)
\end{abstract}

Clusters em fluência semântica consistem em palavras geradas sucessivamente, pertencentes a uma mesma subcategoria, como será especificado abaixo. As subcategorias para roupas/peças de vestuário foram operacionalizadas da seguinte forma: categorias roupas de banho, roupas de festa/social, roupas íntimas, roupas casuais/dia-a-dia, acessórios, roupas de inverno, roupas de verão e calçados. Alguns exemplos de cada subcategoria são listados abaixo, embora possa haver outros.

Pode ocorrer de haver itens pertencentes a duas categorias concomitantemente, enquanto outros deles pertencem exclusivamente a uma categoria e outros a outra. Nestes casos em que ocorre a sobreposição de categorias, os itens sobrepostos são contados em ambas as categorias. Por exemplo, na sequência bermuda, short, camiseta, calça jeans, colete, gravata, paletó,os cinco primeiros itens foram classificados como roupas casuais/dia-a-dia e os três últimos como roupas de festa/social. Neste caso, o item colete foi classificado e pontuado em ambas as categorias, roupas casuais/dia-a-dia e roupas de festa/social.

Nos casos em que clusters menores estiverem dentro de maiores, ou quando duas categorias se sobrepuserem, mas todos os itens podem ser corretamente classificados em uma única categoria, apenas mais comum e a maior delas é pontuada. Por exemplo: na sequencia pulôver, colete, cachecol, manta, touca, blusão, todas são vestimentas de inverno, mas manta e touca poderiam ser classificadas como acessórios, porém mantevese apenas o cluster maior. 


\begin{tabular}{|c|c|}
\hline $\begin{array}{l}\text { Roupas de banho } \\
\text { Peças de vestuário que são utilizadas } \\
\text { em contextos específicos de praia ou } \\
\text { piscina. } \\
\text { Exemplos: roupão, biquíni, maiô, } \\
\text { sunga, canga, touca de banho, saída de } \\
\text { banho. }\end{array}$ & $\begin{array}{l}\text { Roupas de festa/social } \\
\text { Peças de vestuário que são utilizadas em } \\
\text { contextos específicos de festas ou ocasiões } \\
\text { sociais. } \\
\text { Exemplos: vestido longo, longuete, terno, blazer, } \\
\text { paletó, colete, corpete, gravata. }\end{array}$ \\
\hline $\begin{array}{l}\text { Roupas íntimas } \\
\text { Peças de vestuário que são utilizadas } \\
\text { por baixo das peças principais ou em } \\
\text { casa. } \\
\text { Exemplos: calcinha, cueca, sutiã, meia, } \\
\text { anágua, pijama, meia-calça, cuecão, } \\
\text { ceroula. }\end{array}$ & $\begin{array}{l}\text { Roupas casuais/dia-a-dia } \\
\text { Peças de vestuário que são utilizadas no dia-a- } \\
\text { dia, que não implicam um contexto específico. } \\
\text { Exemplos: camisa, camiseta, calça jeans, } \\
\text { bermuda, vestido, moletom, casaco, blusa, } \\
\text { bermuda, saia, abrigo, suéter, corsário. }\end{array}$ \\
\hline $\begin{array}{l}\text { Acessórios } \\
\text { Peças de vestuário que não são as } \\
\text { principais, sendo utilizadas como } \\
\text { acessórios. } \\
\text { Exemplos: gravata, manta, echarpe, } \\
\text { chapéu, touca, luva, boné, gravata, } \\
\text { boina, lenço, avental. }\end{array}$ & $\begin{array}{l}\text { Roupas de inverno } \\
\text { Peças de vestuário que são utilizadas durante a } \\
\text { estação fria do ano, independentemente do } \\
\text { contexto. } \\
\text { Exemplos: calça, casaco de lã, casacão, } \\
\text { sobretudo, blusão de lã, moletom, jaqueta, } \\
\text { japona, suéter, manta, luva, cachecol, touca, } \\
\text { capa de chuva, pala. }\end{array}$ \\
\hline $\begin{array}{l}\text { Roupas de verão } \\
\text { Peças de vestuário que são utilizadas } \\
\text { durante a estação quente do ano } \\
\text { independentemente do contexto. } \\
\text { Exemplos: saia, short, camiseta de } \\
\text { manga curta, regata, vestido, } \\
\text { macaquinho, calção, bata. }\end{array}$ & $\begin{array}{l}\text { Calçados } \\
\text { Peças principais do vestuário dos pés. } \\
\text { Exemplos: sapato, tênis, sandália, chinelo, } \\
\text { rasteirinha, sapatilha, bota, pantufa. }\end{array}$ \\
\hline
\end{tabular}

